\title{
Prediction and Analysis of Abnormal Gas Emission Law in Low- Gas Tunnel Based on K-Line Diagram
}

\author{
Haiyang Wang $\mathbb{D}^{1,2}$ Shulei Zhao, ${ }^{2}$ Jialiang Liu, ${ }^{1,2}$ Yanmin Zhou, ${ }^{2}$ Xiang Chen, ${ }^{2}$ Jie Wang, \\ and Liang Cheng $\mathbb{B D}^{3,4}$ \\ ${ }^{1}$ State Key Laboratory Breeding Base of Mountain Bridge and Tunnel Engineering, Chongqing Jiaotong University, \\ Chongqing 400074, China \\ ${ }^{2}$ School of Civil Engineering, Chongqing Jiaotong University, Chongqing 400074, China \\ ${ }^{3}$ China Merchants Chongqing Communications Technology Research \& Design Institute Co., Ltd., Chongqing 400067, China \\ ${ }^{4}$ State Key Laboratory of Coal Mine Disaster Dynamics and Control, Chongqing University, Chongqing 400044, China
}

Correspondence should be addressed to Liang Cheng; chengliang@cqu.edu.cn

Received 28 August 2020; Accepted 21 September 2020; Published 10 October 2020

Academic Editor: Bin-Wei Xia

Copyright ( 2020 Haiyang Wang et al. This is an open access article distributed under the Creative Commons Attribution License, which permits unrestricted use, distribution, and reproduction in any medium, provided the original work is properly cited.

\begin{abstract}
The risk of gas disaster in the low-gas tunnel is easy to be ignored. By tracking and analyzing the gas monitoring data of the low-gas tunnel, it is found that the cyclic abnormal gas emission occurred many times during the construction period, leading to local gas accumulation, which greatly increases the risk of gas explosion accidents. To scientifically predict the abnormal gas emission in lowgas tunnels, the idea of K-line diagram-based prediction of abnormal gas emission in low-gas tunnels is put forward, and in combination with the field monitoring data of low-gas tunnel (Huangguashan Tunnel), the prediction results with different prediction indexes of K-line diagram are compared and analyzed. The results show that the K-line diagram can reflect the changing trend of gas concentration in real time accurately and show the change law of gas concentration during different construction processes; the moving average (MA) of the K-line diagram can accurately reflect the time of abnormal gas emission, the moving average convergence divergence (MACD) index can reflect the upward or downward power and trend of gas, and the Bollinger Band (BOLL) index can reflect the fluctuation range of gas concentration. The research results can provide a reference for the prediction and prevention of abnormal gas emission in low-gas tunnels.
\end{abstract}

\section{Introduction}

Gas is one of the important disaster factors of gas tunnels. The gas emission quantity and gas content of exposed coal seam in low-gas tunnels are low, which leads to the risk of gas disaster easily underestimated during the construction period. In case of abnormal gas emission during low-gas tunnel construction, local gas overrun and accumulation will easily occur, which will greatly increase the risk of gas explosions and other disasters, and seriously threaten the safety of tunnel construction [1-3]. There are frequent reports of gas accidents in low-gas tunnels. On February 24, 2015, gas explosions occurred in No.1 low-gas tunnel in Luodai Ancient Town, Chengdu, China, due to abnormal gas emission, resulting in 2 deaths and 22 injuries [4]. Scientific pre- diction of abnormal gas emission in low-gas tunnels can provide important early-warning information for gas disaster prevention and control and then help to take targeted measures to intervene and reduce the probability of gas explosion and other disaster accidents. However, the changes in the occurrence characteristics, gas content, gas pressure, geological structure, and other factors of the tunnel-exposed coal seam have a complex nonlinear effect on gas emission, which brings great difficulties to the prediction of abnormal gas emission [5-8]. In the 1980s, Russian scholars proposed for the first time that gas emission should be predicted during coal mining; Greedya [9], a British scholar, initiated the Airey method to predict the gas emission in coal mines based on time and mining technology; Dong [10] proposed gas emission time series method and used it as the regression function 
to establish the Gaussian process regression model, the prediction results of which are accurate and reliable; Liang et al. [11] proposed that, under the condition of considering gas emission source and fluid-solid coupling process, it would be more accurate to predict coal mine gas emission by establishing a dynamic gas prediction model; Booth et al. [12] believe that the limitations of existing gas emission prediction can be solved by the prediction results obtained from improved spatial data sets and the technology including basic physics and energy-related principles. The above research methods are often used to predict the gas emission in coal mines, but there are little research on abnormal gas emission in gas tunnels.

The change in tunnel gas concentration is characterized by fluctuation variability, continuity, and real time [13]. The K-line diagram based on time series can not only weaken the influence of objective factors such as geological conditions and external disturbances but also scientifically and reasonably analyze the gas monitoring information and identify the hidden gas disaster characteristics. More and more researchers have recognized the advantages of the K-line diagram in massive data processing, derivation of related indexes, clear and intuitive trend laws, etc. The introduction of the K-line diagram analysis method into tunnel gas data analysis allows us to analyze and study the changing trend of gas concentration from different angles, which is helpful to the study of gas emission law. Therefore, this paper puts forward the idea of the K-line diagram-based prediction of abnormal gas emission in low-gas tunnels and forecasts and analyzes the gas emission in low-gas tunnels, to reduce the possibility of gas disasters.

\section{Introduction and Characteristic Analysis of K-Line Diagram}

2.1. Introduction of K-Line Diagram. K-line diagram, also known as the candlestick chart, can be traced back to the era of the Tokugawa Shogunate in the 18th century in Japan $[14,15]$. It is three dimensional and intuitive with a unique method covering comprehensive records and containing large information content, which is widely used in stock, futures, foreign exchange, options, and other securities markets. Like the stock market, the change of tunnel gas concentration is also affected by many factors under the internal and external complex environments. Therefore, it is a promising means to introduce the K-line diagram method into the cause diagnosis and early-warning analysis of tunnel gas anomaly and to make use of its advantages in processing and analyzing data. By analyzing the theoretical basis of K-line and the meaning of K-line shapes for tunnel gas monitoring and extending them to the characterization method of gas anomaly patterns [16], it can provide a new research idea for the field of gas data monitoring and early warning.

The traditional K-line diagram usually revolves around four data of the K-line-the opening, the highest, the lowest, and the cut-off prices-thus reflecting the trend and price of stocks. When it is applied to gas early warning, a single K-line diagram can reflect the changing trend of gas emission concentration in a unit time. When combined with other techni- cal indexes, it can also predict the change direction and trend of gas emission intensity in the future. The K-line diagram has different unit times, such as minute K-line, hour K-line, and day K-line. In this paper, the initial gas concentration $\mathrm{O}$, the highest concentration $\mathrm{H}$, the lowest concentration $\mathrm{L}$, and the cut-off concentration $\mathrm{C}$ are taken as the four data of the K-line diagram. In the traditional K-line diagram, the red line represents the upward trend of gas concentration in one cycle, and the green line represents the downward trend of gas concentration in one cycle. The specific parameters are shown in Figure 1.

The K-line diagram contains four elements: color, entity size, shadow length, and unit time. Therefore, when it is applied to the time series of tunnel gas concentration, the trend of gas concentration contained in it can be found out; thus, the time point at which the abnormal gas concentration occurs can be captured more accurately. The main function of the K-line diagram is to make a reasonable judgment according to its K-line shape. The process of understanding the K-line diagram is mainly to observe the red line, green line, entity size, and shadow length, taking the red line as an example, as shown in Table 1.

\subsection{Common Characteristics of K-Line Diagram and Gas} Concentration Change Curve. The change of tunnel gas concentration shares the following common characteristics with the stock market, so the use of the K-line diagram to describe gas concentration change has a theoretical basis.

\section{(1) Fluctuation variability}

The essence of K-line is to reflect the fluctuation of commodity value caused by the natural law of market supply and demand and manmade speculation, while the assessment of gas disaster risk is mainly to record and analyze the gas concentration and to properly display the geological conditions and artificial disturbance in the tunnel site area through the change of gas concentration, so the change of gas concentration state also has two influencing factors: objective naturality and external factors.

\section{(2) Continuity}

Both the market price changes recorded by K-line and the monitoring results by existing gas concentration monitoring equipment are continuous records. Through the existing instruments and equipment, long-time, continuous and uninterrupted data collection can be achieved to provide necessary conditions for judging the change of gas concentration.

\section{(3) Real time}

Real-time acquisition of stock market data enables investors to obtain market dynamic changes more quickly. Similarly, the real-time change of gas concentration is very important for assessing the current gas disaster risk and adjusting the construction ventilation scheme. With the development of science and technology, the indicators for assessing the risk of gas disasters have developed from 


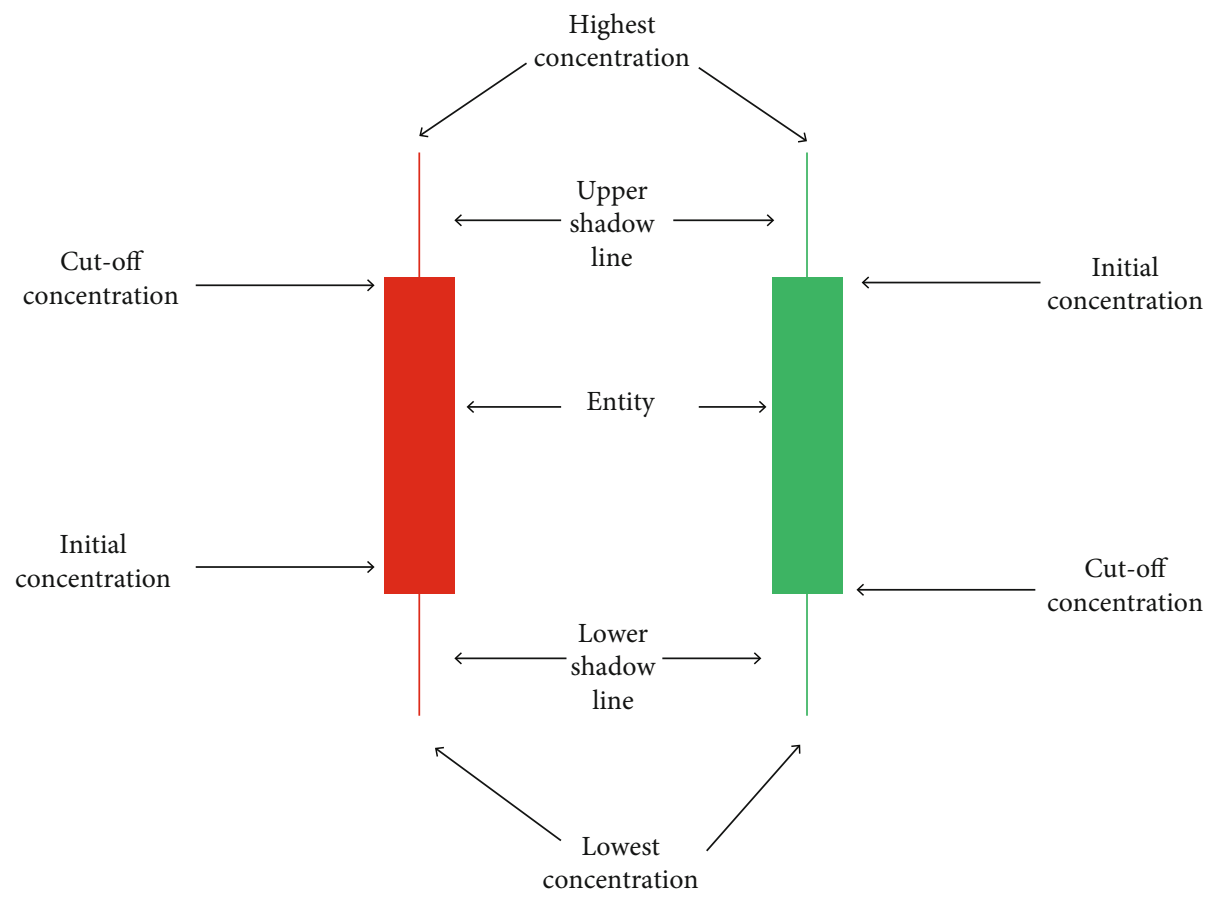

Figure 1: Sketch diagram of K-line.

TABLE 1: Graphics of the K-line diagram and their meanings.

\begin{tabular}{|c|c|c|c|c|c|}
\hline \multicolumn{6}{|l|}{ Graph } \\
\hline Name & Short red line & $\begin{array}{c}\text { A red entity } \\
\text { with long lower } \\
\text { shadow }\end{array}$ & $\begin{array}{l}\text { A red entity with } \\
\text { upper and lower } \\
\text { shadows }\end{array}$ & A red entity with lower shadow & $\begin{array}{l}\text { A red entity with an } \\
\text { upper shadow }\end{array}$ \\
\hline Meaning & $\begin{array}{l}\text { Indicating that the } \\
\text { growth trend in the cycle } \\
\text { is not obvious, which } \\
\text { needs further } \\
\text { observation }\end{array}$ & $\begin{array}{l}\text { Indicating that } \\
\text { the gas } \\
\text { concentration } \\
\text { increases in the } \\
\text { cycle }\end{array}$ & $\begin{array}{l}\text { Indicating that it will } \\
\text { jump, open high, and } \\
\text { go low in the next } \\
\text { period of the cycle }\end{array}$ & $\begin{array}{l}\text { Indicating that the gas concentration } \\
\text { may increase in the cycle }\end{array}$ & $\begin{array}{l}\text { Indicating that the } \\
\text { gas concentration in } \\
\text { the cycle has a strong } \\
\text { growth momentum }\end{array}$ \\
\hline Graph & & & & \begin{tabular}{l|l} 
\\
\end{tabular} & \\
\hline Name & $\begin{array}{l}\text { A red entity without a } \\
\text { shadow }\end{array}$ & Inverted T-line & T-line & Cross & \\
\hline Meaning & $\begin{array}{l}\text { Indicating a strong } \\
\text { growth in the next period } \\
\text { of the cycle }\end{array}$ & $\begin{array}{l}\text { Indicating that } \\
\text { the cycle may } \\
\text { be shortened }\end{array}$ & $\begin{array}{l}\text { Indicating that the } \\
\text { cycle may expand }\end{array}$ & $\begin{array}{l}\text { Indicating a peak signal or a bottom } \\
\text { signal, which indicates that, in the } \\
\text { cycle, the trend of the gas } \\
\text { concentration interval will change or } \\
\text { it will enter a plateau }\end{array}$ & \\
\hline
\end{tabular}

subjective judgment and manual acquisition to objective quantitative evaluation of equipment detected physiological indexes, and from the index parameter acquisition in a cer- tain period to current real-time recording of continuous parameters, which also provides conditions for complete and instant monitoring of gas concentration changes. 


\section{K-Line Diagram of Low-Gas Tunnel Abnormal Gas Emission}

3.1. Overview of Huangguashan Tunnel. Huangguashan Tunnel is located in Yongchuan District, Chongqing, which is constructed by the drilling-and-blasting method. It is an extralong tunnel, with a total length of $3268 \mathrm{~m}$. The coal measure stratum that the tunnel passes through is the 5th member of Xujiahe Formation, which only contains coal locally, and belongs to the extremely thin coal seam. The thickness of the coal seam is unstable, and even pinch out occurs. It is a typical low-gas tunnel. The construction can be divided into the construction processes of drilling and blasting, mucking, frame erecting, and shotcreting. Through longterm tracking of the gas concentration change data of Huangguashan Tunnel, it was found that there was gas continuously emitting into the tunnel in each construction process during the construction. The difference was that the gas concentration emitting into the tunnel is different in different processes. Through the study of the relationship between gas concentration and construction processes, the gas concentration emitting into the tunnel during each construction process can be distinguished. The changes in gas concentration based on the data of gas concentration at the tunnel face in one construction cycle are shown in Table 2 and Figure 2.

From the comparative analysis of Figures 2 and 3, it can be seen that (1) there is an abnormal point of gas concentration higher than the highest point of the box diagram, which indicates that the gas concentration fluctuates greatly and there is abnormal gas emission, and (2) the gas concentration during the construction period is generally maintained between $0.00 \%$ and $0.1 \%$, but during the blasting operation, the gas concentration will increase to $5 \%$. So, it can be seen that the blasting operation is the main cause of abnormal gas emission in tunnels.

3.2. K-Line Diagram of Gas Concentration. The tunnel gas monitoring system will record the change of gas concentration in real time. If the selection period is too small, the data will be disordered and not intuitive; if the selection period is too large, it cannot timely describe the abnormal gas emission. After a large number of experiments, the period of $10 \mathrm{~min}$ is most appropriate. So, in this paper, taking $10 \mathrm{~min}$ as a cycle, a K-line of gas concentration in one construction cycle is drawn by using Origin and Excel software jointly and improved, as shown in Figure 4. The traditional K-line diagram has only two colors to express the change in stock price. When it is applied to the change of tunnel gas concentration, it can only express the increase or decrease of gas concentration in different cycles, but it is not accurate enough. For this purpose, this paper innovatively introduces a three-color K-line diagram. The yellow line is the new red line, representing an increase in gas concentration; the green line represents the decrease of gas concentration; when the gas concentration is greater than $0.5 \%$, the K-line diagram of the gas concentration becomes eye-catching red to remind the construction personnel of the gas overrun. At the same time, when the gas concentration of the adjacent K-line dia- gram changes greatly, the K-line diagram is transformed into the corresponding gradient color. The improved three-color $\mathrm{K}$-line diagram has the following advantages over the traditional K-line diagram:

(1) The color-gradient line represents abnormal gas emission and is named as "sharply-increasing line." The moment of abnormal gas emission can be accurately determined by the position of "sharplyincreasing line"

(2) The gas concentration at $0.5 \%$ is set as a warning line. When the gas concentration is greater than $0.5 \%$, both the "yellow line" and "green line" turn red, which can express the situation of gas concentration overrun more intuitively

In Figure 4, except for the small fluctuation of gas concentration in the blasting stage, the gas concentration in other stages is consistent with the actual situation in the previous section, indicating that it is feasible to express the change of gas concentration with the K-line diagram.

To express the change of gas concentration more accurately, the concept of "K-line coordinates" is introduced, and the position matching issue between $\mathrm{K}$-lines is solved by defining the coordinates of K-lines in the sequence. The order of $\mathrm{K}$-lines in the K-line sequence is called the $\mathrm{K}$-line abscissa, the increasing degree of the gas cut-off concentration in each cycle relative to that in the previous cycle is called the K-line ordinate, with the ordinate of the first K-line in the K-line sequence set to 1, as shown in Figure 5. By analyzing the increasing degree, the moment that the abnormal gas concentration occurs can be obtained intuitively and clearly, which is consistent with the actual situation.

\section{Application of K-Line Diagram Basic Indexes in Prediction of Low-Gas Tunnel Gas Emission Law}

It is too one sided to judge the gas emission trend by using the $\mathrm{K}$-line diagram only. The K-line combinatory analysis of gas emission trends by MA, MACD, and BOLL indexes is more reasonable.

4.1. Moving Average (MA). Using MA to calculate the arithmetic moving average of the monitored gas concentration value in a cycle and draw it into a line can directly reflect the trend of gas emission. The calculation formula of MA is as follows:

$$
\operatorname{MA}(X, N)=\frac{X_{1}+X_{2}+. . \cdots+X_{N}}{N},
$$

where MA is the moving average, $X_{i}$ is the end value in the unit time, and $N$ is the calculation cycle.

There is no fixed rule for the choice of long-cycle and short-cycle MAs. The intersection of the long-cycle average and short-cycle average represents the development trend of gas concentration. The short-term average can closely follow the development trend of gas concentration and can 
TABLE 2: Gas concentration in different construction processes at the tunnel face.

\begin{tabular}{lccccc}
\hline Construction process & Time $(\mathrm{h})$ & Gas concentration (\%) & Construction process & Time (h) & Gas concentration (\%) \\
\hline \multirow{4}{*}{ Drilling and blasting } & 0 & 0.02 & & 8 & 0.06 \\
& 1 & 0.02 & Frame erecting & 10 & 0.05 \\
& 2 & 0.47 & & 11 & 0.04 \\
& 3 & 0.35 & & 12 & 0.04 \\
Mucking & 4 & 0.26 & Shotcreting & 13 & 0.06 \\
& 5 & 0.04 & & 14 & 0.01 \\
& 6 & 0.02 & & 15 & 0.04 \\
\hline
\end{tabular}



FIGURE 2: Box plot of gas concentration in different construction processes.

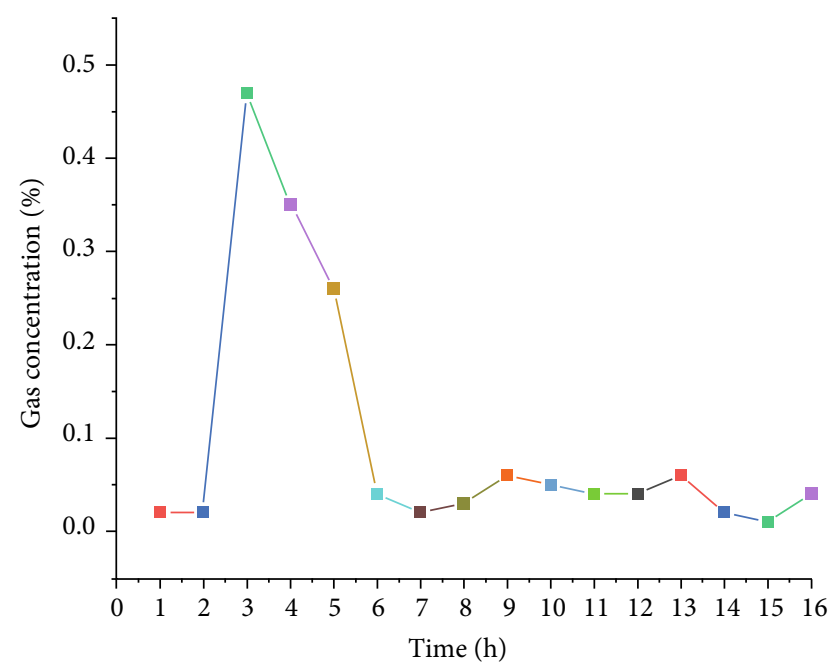

FIgURE 3: Gas concentration over time series.

better represent the gas change in the short term; while the long-term average is less affected by short-term gas concentration fluctuation, which is more stable and can well reflect the long-term trend. Set the black line and red line in Figure 6 as MAs with the calculation cycles of $0.5 \mathrm{~h}$ and $1 \mathrm{~h}$, respectively.

It can be seen from Figure 6 that, when the short-term average $(0.5 \mathrm{~h})$ crosses the long-term average $(1 \mathrm{~h})$ upwards, this breakthrough point is called "golden cross." When the "golden cross" appears, the gas concentration shows an upward trend in the short term. When the short-term average falls below the long-term average, the breakthrough point is called "death crossing," and the gas concentration shows a downward trend in the short term. The MA shows strong regularity during the normal gas fluctuation stage, and MA starts to rise in the blasting stage, then reaches the peak value, and then falls back immediately after the end, which is in line with the actual situation.

There is a lag phenomenon in the MA signal, but the trend can often last for a while. Therefore, when MA sends a signal that the gas emission increases or decreases, although the current gas emission may still be in a stable trend, it still indicates that the next gas emission may change significantly. Therefore, timely gas prevention measures can be taken according to the signal at this time. Moving average is used in the calculation of the next two types of indexes.

4.2. Moving Average Convergence Divergence (MACD) Index. Moving average convergence divergence index is referred to as the MACD index for short, which is aimed at revealing the changes in the intensity, direction, momentum, and duration of the gas trend, and has a certain guiding role in preventing gas concentration overrun. MACD index is a collection of three-time series calculated from historical data, which are MACD series itself, "average" series, and "divergence" series. MACD series is the DIF of the fast MA (EMA1) and slow MA (EMA2) and the difference (DIF) between EMA1 and EMA2. The average series is composed of $N$-cycle moving average convergence divergence (DEA) of DIF. Divergence is the difference between the MACD series and the "average" series, which is usually displayed as a bar graph, and its length is twice that of DIF-DEA. Therefore, the MACD index includes two lines and one stick; the fast line is DIF, and the slow line is DEA, as shown in Figure 7. 


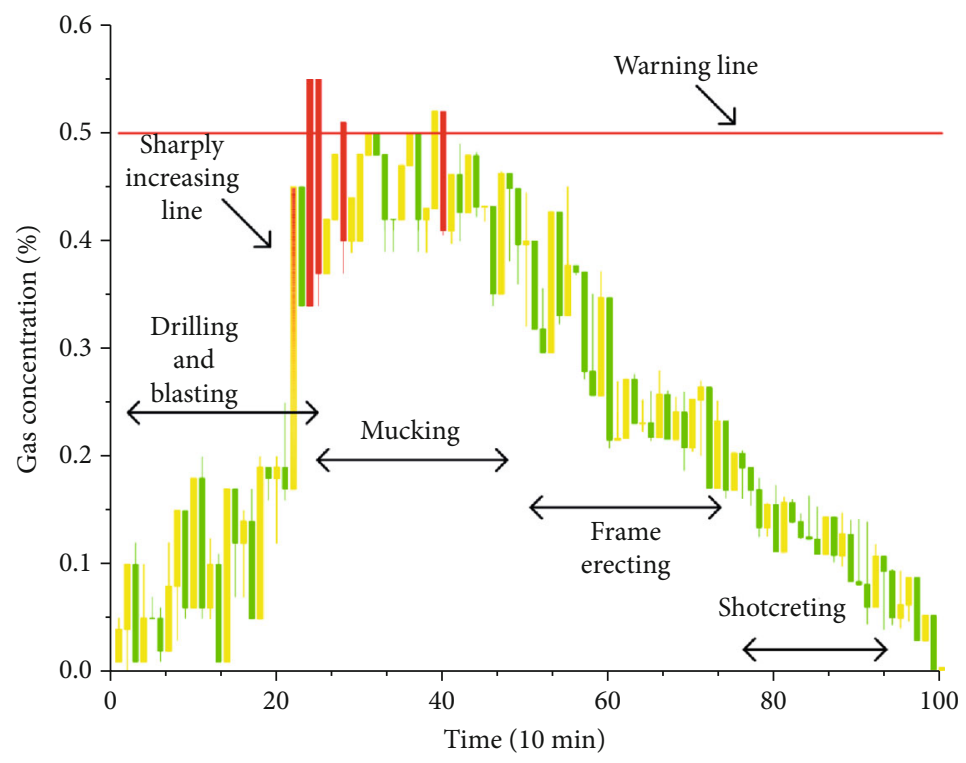

FIgURE 4: K-line diagram of gas concentration.
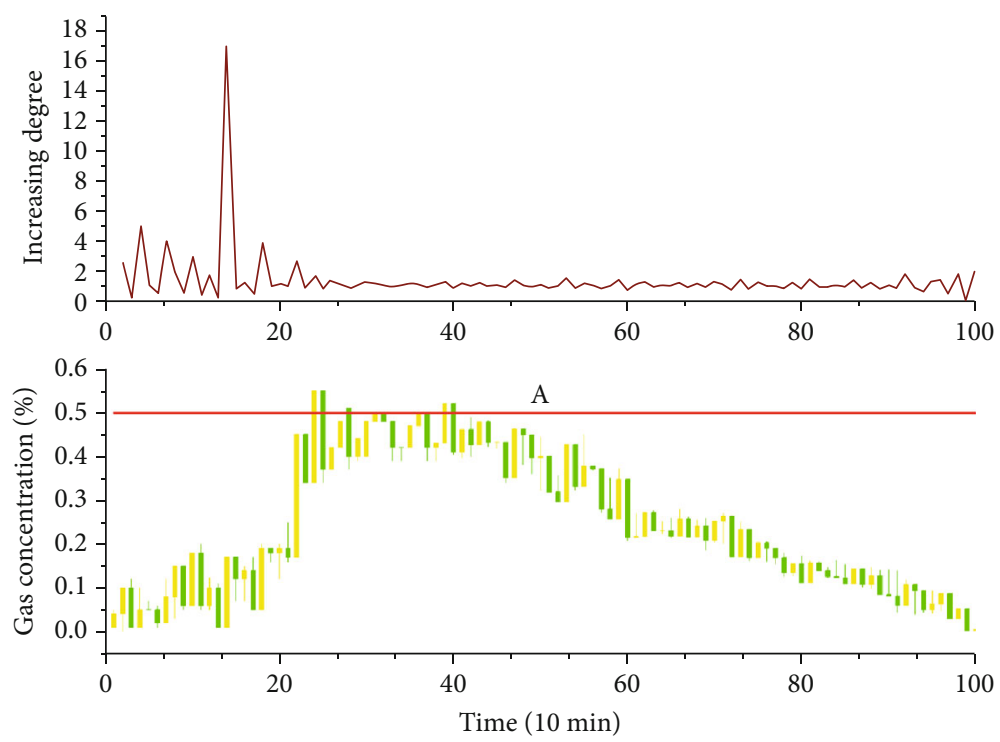

FIGURE 5: Increase degree of gas concentration.

The calculation formula is as follows:

$$
\mathrm{EMA}_{T}(12)=\frac{2}{12+1} \times C(T)+\frac{11}{12+1} \times \mathrm{EMA}_{T-1}
$$

where $\mathrm{EMA}_{T}(12)$ is today's fast-moving MA, $C(T)$ is today's gas cut-off concentration, and $\mathrm{EMA}_{T-1}$ is yesterday's fastmoving MA.

$$
\mathrm{EMA}_{T}(26)=\frac{2}{26+1} \times C(T)+\frac{25}{26+1} \times \mathrm{EMA}_{T-1} \text {, }
$$

where $\mathrm{EMA}_{T}(12)$ is today's slow-moving $\mathrm{MA}, C(T)$ is today's gas cut-off concentration, and $\mathrm{EMA}_{T-1}$ is yesterday's slow-moving MA.

$$
\begin{gathered}
\text { DIF }=\operatorname{EMA}(12)-\operatorname{EMA}(26), \\
\operatorname{DEA}(\text { MACD })=\frac{2}{10} \times \mathrm{DIF}_{T}+\frac{8}{10} \times \mathrm{DEA}_{T-1},
\end{gathered}
$$

where DEA $_{T-1}$ is yesterday's DEA.

In Figure 7 , the blue bar graph represents twice DIFDEA, the black line is slow line DEA, and the red line is fast line DIF. When applying the MACD index, it is mainly to analyze the relationship between DIF and 0 axis, DIF and DEA, and the sticks and 0 axis. When the bar graph is above the 0 axis, it means that the gas concentration is rising, and the higher the bar graph, the greater the upward amplitude 


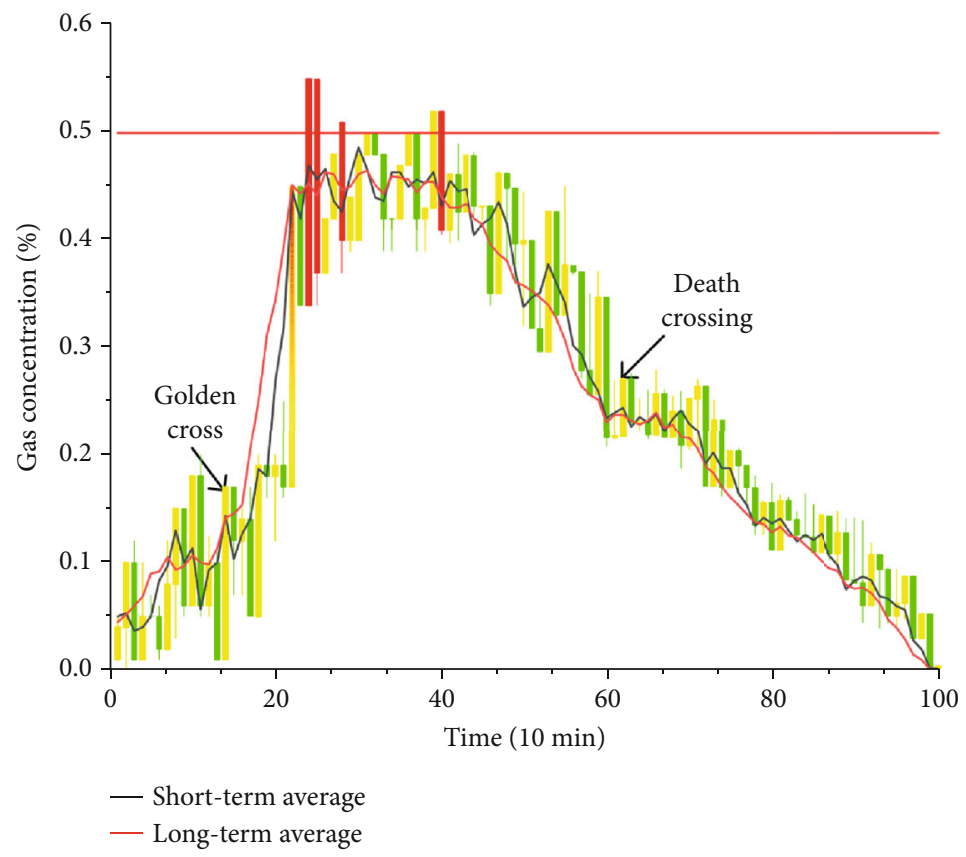

FIGURE 6: MA diagram of gas concentration.
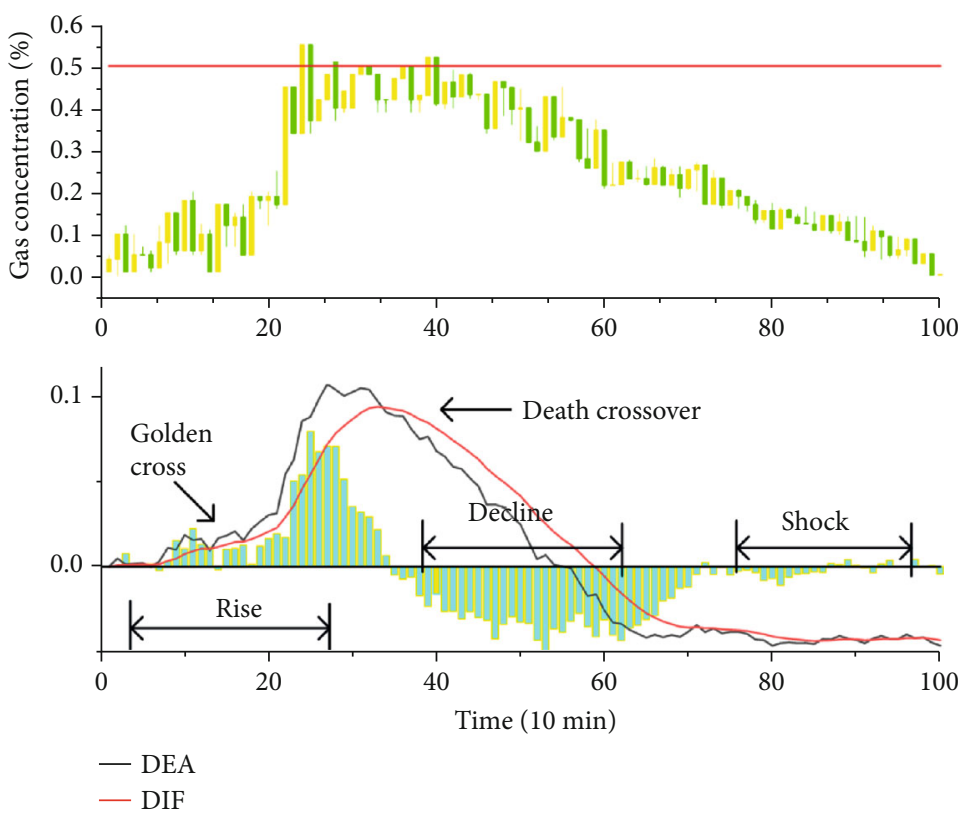

Figure 7: MACD index of gas concentration.

and the stronger the upward power. If the bar graph is below the 0 axis, it means that the gas concentration is in a downward trend. This index can intuitively indicate the degree of gas concentration increase in the blasting stage and the degree of gas concentration decrease when the blasting stage ends and enters the supporting stage, and the corresponding sticks have increased or decreased, thus quantifying the magnitude of the change. When the black line (slow line DEA) crosses the red line (fast line DIF) upwards, a "golden cross" appears. At this time, the bar graph is above the 0 line and its height represents the increased amplitude. When the gray line crosses the red line downward, a "death crossing" appears. At this time, the bar graph is below the 0 line and the height of the bar graph represents the decline amplitude. Compared with the "golden cross" of MA, the golden cross signal of the MA index indicates that the short-term gas concentration exceeds the long-term gas concentration, which is a signal that the whole gas concentration is about to increase. The "golden cross" signal of the MACD index indicates that the decreased speed of the gas concentration is slowing down, 
or the increased speed is accelerated, which also proves that the gas concentration will increase in the future. If the golden cross signals of the two indexes appear at the same time, or almost at the same time, they play a role of mutual verification. It shows that the gas concentration not only shows an increasing trend but also will increase faster and faster in the future. When such signals appear, it can be determined that the gas concentration will increase in a period in the future, so we should pay close attention to its increased amplitude and changing trend.

According to these characteristics of MACD, it can be determined that in tunnel gas monitoring, when DIF crosses DEA and breaks through DEA from bottom to top in MACD index, it indicates that the monitoring value will rise; when DIF breaks through from bottom to top, it means that the monitoring value will rise; if DIF line continues to rise, it means that the monitoring value will be in danger of exceeding the limit in a period in the future, and corresponding preventive measures should be taken at this time. MACD index can also express the convergence and divergence of the exponential moving average (EMA), which uses the speed difference between short cycle and long cycle to express the comparison trend between the gas concentration compared to the current cycle and the average value of the last cycle. When the gas concentration is in a state of fluctuation, the difference between the short cycle and the long cycle will be small. If the MACD value continues to increase at this time, the divergence will continue to expand.

4.3. Bollinger Band (BOLL) Index. The technical index of BOLL in the K-line diagram can be automatically adjusted with the change of gas concentration, and it is more flexible and intuitive than other methods. Bollinger Band is a technical index designed according to the standard deviation principle in statistics. The index consists of three track lines. In the field of gas early warning, the upper and lower lines in this index can be regarded as the pressure line and support line of the gas index, respectively. Between the two lines, there is an average line of a gas index. Generally, the gas index will wander within the band-shaped interval composed of the upper and lower tracks, which can be called the reasonable range of gas concentration, and the position of the track can be automatically adjusted with the change of numerical value. When the band narrows, the gas index may fluctuate violently, as shown in Figure 8. BOLL index includes three curves, namely, the upper track, the middle track, and the lower track, where the middle trajectory is the moving average, denoted as $u_{n}$.

The calculation method of the upper track is

$$
\overline{\mathrm{Bn}}=u_{n}+\alpha \times \sigma_{n} .
$$

The calculation method of the lower track is

$$
\begin{gathered}
\overline{\mathrm{Bn}}=u_{n}-\alpha \times \sigma_{n}, \\
\sigma_{n}=\sqrt{\frac{1}{n} \sum_{i=1}^{n}\left(X_{i}-u_{n}\right)^{2},}
\end{gathered}
$$

where $\alpha$ is a multiple of standard deviation, which is generally 2 by default.

The moving range of the channel formed by the upper, middle, and lower tracks in the BOLL index is uncertain, and the upper and lower limits of the channel change with the fluctuation of gas concentration. Under normal circumstances, the gas concentration K-line should always run on the channel. If the gas concentration exceeds the channel range, it means that the gas concentration in the working face is in an overrun state, and the construction should be stopped immediately. In the BOLL index, the upper and lower tracks of the channel indicate the highest and lowest positions of gas concentration in a certain period. Generally speaking, when the gas concentration runs above the middle track of BOLL, it indicates that the gas concentration is in a strong upward trend; when the gas concentration runs below the middle track of BOLL, it indicates that the gas concentration is in a strong downward trend. Under normal conditions, the gas index usually runs within a band a certain width, and its characteristic is that the index value does not appear to suddenly increase or decrease, in a relatively balanced state. When the gas index crosses the upper limit pressure line, it is an upward signal; when the index crosses the lower limit support line, it is a downward signal; when the index crosses the middle boundary line from bottom to top, it means that it may rise continuously for some time; when the gas concentration crosses the middle boundary line from top to bottom, it means that it may decline continuously for a while.

BOLL has three functions in gas early warning: (1) BOLL can indicate the allowable maximum and minimum values of gas index, (2) BOLL can show the trend of gas concentration, and (3) Bollinger Bands are usually used as an auxiliary index to judge the trend, that is, to evaluate the strength of the gas index trend by the position of the gas index in the Bollinger Bands.

4.4. Technical Indexes of K-Line Diagram and Verification. To avoid contingency, the original abnormal fluctuation data of gas concentration is increased from 100 groups to 200 groups for trend analysis and prediction. The time series diagram is shown in Figure 9, and the K-line and technical indexes of gas concentration are shown in Figure 10.

From the comparative analysis of Figures 9 and 10, it can be seen that the overall trend of the K-line diagram is the same as that of the time series diagram, and the abnormal emission of gas concentration can be accurately expressed in combination with the increasing degree. As for the moving average, according to Figure 10, when the overall gas concentration reaches the peak, the corresponding short-term moving average is higher than the long-term moving average, so the gas concentration shows an upward trend in this period. After reaching the peak, the long line and short line immediately fell back, and the falling speed of the short-line was faster than that of the long-line, which proved that the gas concentration shows a downward trend. 


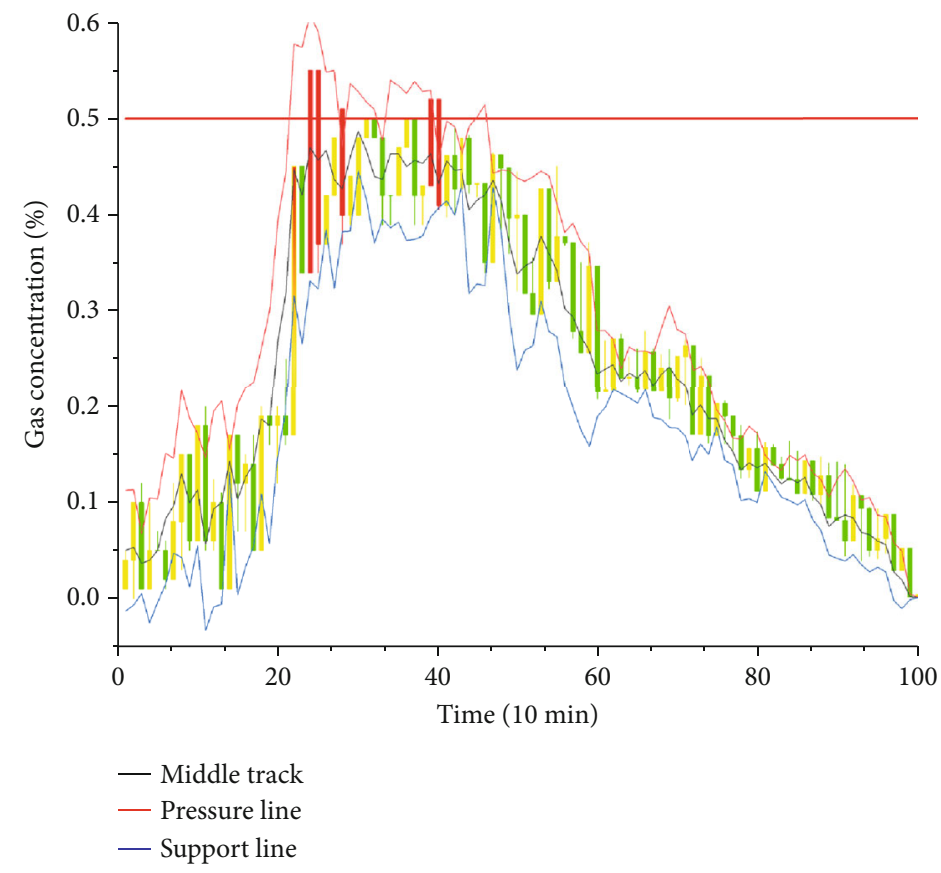

FIGURE 8: BOLL of gas concentration.



Figure 9: Time series diagram.

For the MACD index, when the gas concentration rises to the peak, the corresponding bar graph is above the 0 axis and reaches the highest value. When the gas concentration falls back, the bar graph is below the 0 axis and changes from high to low, which proves that the gas concentration drops. This shows that the corresponding sticks have changed obviously, thus quantifying the amplitude of variation. As shown in Figure 10, DIF crosses DEA and breaks through DEA from bottom to top in the MACD index, which indicates that the monitoring value is rising, and DIF is gradually increasing and continuously rising upwards, indicating that the gas monitoring value is in danger of exceeding the limit at this time. At this time, the moving average and MACD index has a "golden cross," so it can be determined that the gas con- centration is in a strong upward stage at this time, which is consistent with the actual gas trend.

For the BOLL index, when an abnormal gas concentration emission occurring, the difference between the middle track and the upper track is small, and when the middle track breaks through the upper track, it means gas concentration overrun in the working face, so the construction should be stopped immediately.

Based on the above, using the increasing degree, the MA, MACD, and BOLL indexes of gas, the changing trend of gas concentration can be displayed intuitively, and the gas emission state, gas concentration change trend, and whether the gas concentration fluctuates abnormally can be judged according to various technical diagrams and lines.

\section{Conclusions}

(1) The amplitude and fluctuation of gas emission concentration in the tunnel construction operation cycle can be described by the K-line diagram. The improved K-line diagram is easy to read, practical, and effective, and the actual change of gas concentration can be observed more comprehensively and thoroughly. A series of other indexes are derived based on this diagram to describe the law of gas emission, which can better predict the trend of abnormal gas emission in low-gas tunnels

(2) MA index is used to reflect the long-term change trend or cycle of gas emission concentration; the MACD index is used to reflect the intensity, direction, energy, and trend cycle of gas emission concentration change; BOLL is used to reflect the possible 


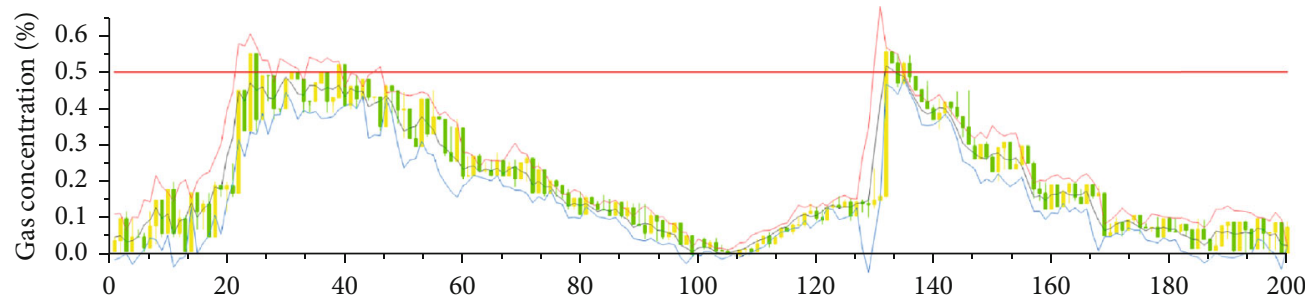

— Middle track

- Upper track

- Lower track
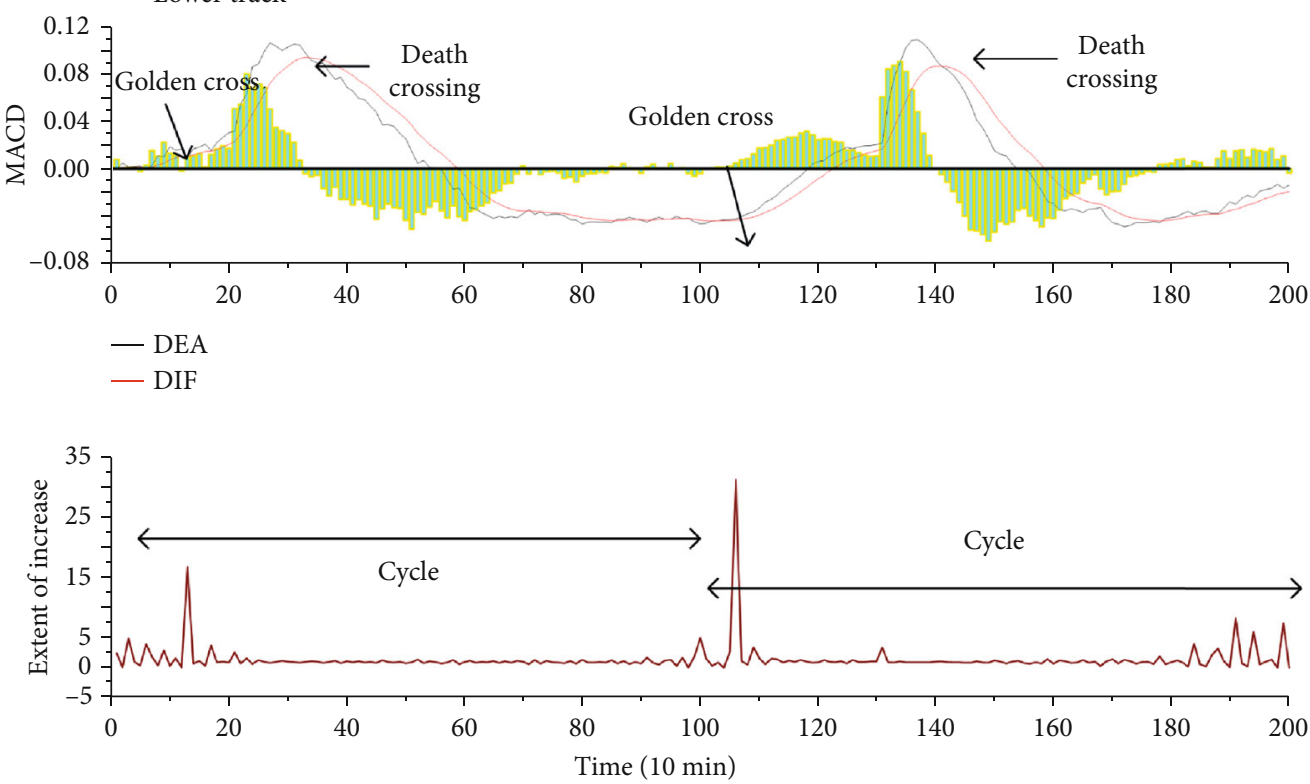

Figure 10: K-line and technical indexes of gas concentration.

upper and lower limits of gas emission. The abnormal gas emission law judged in combination with the K-line diagram and its indexes is consistent with the actual situation

(3) The K-line diagram and its indexes can not only be used to describe the change law of gas concentration in low-gas tunnels but also provide ideas for describing the change of gas concentration in high-gas tunnels and mines

\section{Data Availability}

The data used to support the findings of this study are included within the article.

\section{Conflicts of Interest}

The authors declare that they have no conflicts of interest.

\section{Acknowledgments}

This paper is jointly funded by the National Natural Science Foundation of China (No. 51804058 and No. 51804055), the science and technology research plan of Chongqing Education Commission (Grant No. KJQN201800729), and the sci- ence and technology innovation project (2020S0022) of Chongqing Jiaotong University.

\section{References}

[1] M. Deng, G. S. Zhang, and Q. H. Chen, "Forecast of coal and gas outburst based on time series of gas concentration," Journal of China Coal Society, vol. 35, no. 6, pp. 260-263, 2010.

[2] J. H. Fu and Y. P. Cheng, "Situation of coal and gas outburst in China and control counter measures," Journal of Mining \& Safety Engineering, vol. 24, no. 3, pp. 253-259, 2007.

[3] W. Liang, Research on mine gas anomaly diagnosis and early warning based on K-line principle, China University of Mining and Technology, 2017.

[4] J. Zhou, Research on disaster early warning method based on gas emission law of working face, Xi'an University of science and technology, 2019.

[5] T. Liu, B. Q. Lin, W. Yang, T. Liu, and C. Zhai, “An integrated technology for gas control and green mining in deep mines based on ultra-thin seam mining," Environmental Earth Sciences, vol. 76, no. 6, pp. 243-259, 2017.

[6] K. Wang, J. Zang, G. Wang, and A. Zhou, "Anisotropic permeability evolution of coal with effective stress variation and gas sorption: model development and analysis," International Journal of Coal Geology, vol. 130, pp. 53-65, 2014. 
[7] Z. Pan, Y. Ma, L. D. Connell, D. I. Down, and M. Camilleri, "Measuring anisotropic permeability using a cubic shale sample in a triaxial cell," Journal of Natural Gas Science and Engineering, vol. 26, pp. 336-344, 2015.

[8] W. Li and Z. H. Han, "Application of improved grey prediction model for power load forecasting," in International Conference on Computer Supported Cooperative Work in Design, Xi'an, China, April 2008.

[9] D. P. Greedya, "Geological controls on the formation and distribution of gas in British coal measure strata," International Journal of Coal Geology, vol. 10, no. 1, pp. 1-31, 1988.

[10] D. W. Dong, "Mine gas emission prediction based on Gaussian process model," Procedia Engineering, vol. 45, no. 2, pp. 334338, 2012.

[11] L. Chen, E. Wang, J. Feng, X. Kong, X. Li, and Z. Zhang, "A dynamic gas emission prediction model at the heading face and its engineering application," Journal of Natural Gas Science and Engineering, vol. 30, pp. 228-236, 2016.

[12] P. Booth, H. Brown, J. Nemcik, and R. Ting, "Spatial context in the calculation of gas emissions for underground coal mines," International Journal of Mining Science and Technology, vol. 27, no. 5, pp. 787-794, 2017.

[13] T. H. Lu and Y. M. Shiu, "Tests for two-day candlestick patterns in the emerging equity market of Taiwan," Emerging Markets Finance \& Trade, vol. 48, no. sup1, pp. 41-57, 2014.

[14] Y.-J. Goo, D.-H. Chen, and Y.-W. Chang, "The application of Japanese candlestick trading strategies in Taiwan," Investment Management and Financial Innovations, vol. 4, no. 4, pp. 4979, 2007.

[15] Y. Izumi, T. Yamaguchi, S. Mabu, K. Hirasawa, and J. Hu, "Trading rules on the stock markets using genetic network programming with candlestick chart," in 2006 IEEE International Conference on Evolutionary Computation, Vancouver, BC, Canada, July 2006.

[16] W. W. Y. Ng, X.-L. Liang, P. P. K. Chan, and D. S. Yeung, "Stock investment decision support for Hong Kong market using RBFNN based candlestick models," in International Conference on Machine Learning \& Cybernetics, pp. 538-543, Guilin, China, July 2011. 